\title{
Correction to the Higher Dimensional Black Hole Entropy
}

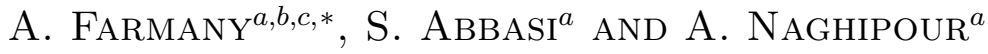 \\ ${ }^{a}$ Department of Chemistry, Ilam University, Pajuhesh str., Ilam, Iran \\ ${ }^{b}$ Young Researchers Club, Azad University of Ilam, Iran \\ ${ }^{c}$ Michigan Center for Theoretical Physics, Michigan University, USA
}

(Received March 5, 2007; revised version March 17, 2008)

\begin{abstract}
Using the modified Hawking radiation of a Schwarzschild black hole (based on the generalized uncertainty principle) we obtained the BekensteinHawking entropy of a higher dimensional Schwarzschild black hole. Furthermore, the thermodynamics of such black hole is studied.
\end{abstract}

PACS numbers: 04.60.-m, 04.70.-s, 04.70.Dy, 03.65.Ud

\section{Introduction}

If our universe is a brane embedded in some higher dimensional space, the hierarchy problem can be solved where the higher dimensional Planck scale is low and the extra dimensions large $[1,2]$. In the brane-world scenario [3-6], gravitational collapse of trapped matter on a brane will produce a black hole. On the other hand, the higher dimensional black hole may be produced at a high energy probe [7]. These black holes are microscopic, comparable in the size to the elementary particles. The production of black holes by particle accelerators is an exciting possibility. When it comes to producing high energies, no device outdoes great accelerators. These machines accelerate subatomic particles to velocities exceedingly close to the speed of light. These particles then have enormous kinetic energies. At the LHC, a proton will reach energy of roughly $7 \mathrm{TeV}$. According to the Einstein special relativity these energies are equivalent to a mass of $10^{-23} \mathrm{~kg}$ when two particles collide at the closed range, their energy is concentrated into a tiny region of space. This types of black hole are microscopic, comparable in size to elementary particles, they could evaporate shortly after they had formed, because the emission carries out the energy, the mass of black hole tends to decrease.

*e-mail: a.farmany@usa.com 
The evaporation of these holes would leave very distinctive imprints on the detectors. Typical collisions produce moderate numbers of high-energy particles, but decaying black hole is different. According to the Hawking works [11], the black hole radiates a large number of particles and the prospect of producing black holes on Earth may strike. How do we know that it would safely decay, as Hawking predicted, instead of continuing to grow, eventually consuming the entire planet? It turns out that general quantum reasoning implies that microscopic black holes cannot be stable and therefore are safe. Concentrations of mass energy such as elementary particles, are stable only if a conservation law is forbidden, their decay is no conservation law to stabilize a small black hole. In quantum theory, anything not expressly forbidden is compulsory, so small black hole will rapidly decay, in accordance with the second law of thermodynamics [8-12]. Indeed, according to quantum mechanics an empirical argument corroborates that black holes would pose no danger. In this letter we calculate the Hawking radiation of higher dimensional black holes by a generalized uncertainty principle. In the canonical quantum gravity the character of the Hawking radiation is modified when quantum gravity effects are properly taken into account, even for non-rotating, neutral and very massive black hole with respect to the Planck scale. Derivation of the usual Hawking radiation is based on the uncertainty principle [11], $\Delta x_{i} \Delta p_{j} \geq \hbar \delta_{i j}$ where $i, j=1, \ldots, d-1$. Derivation of the Hawking radiation of the mini black hole will be based on the generalized uncertainty principle.

\section{Theory}

Let us begin with a Schwarzschild black hole that lives on a $d$-dimensional space-time. In this letter we obtain the black hole radiation in the higher dimensional space. Furthermore, the thermodynamics of such black hole is considered.

A natural candidate for such hole is the $d$-dimensional Schwarzschild solution,

$$
\mathrm{d} s^{2}=\left(1-\theta_{d-2}\right) c^{2} \mathrm{~d} t^{2}-\left(1-\theta_{d-2}\right)^{-1} \mathrm{~d} r^{2}+r^{2} \mathrm{~d} \Omega_{d-2},
$$

where

$$
\theta_{d-2}=\frac{16 \pi G_{d} M}{(d-2) \Omega_{d-2} c^{2} r^{d-3}}
$$

and $G_{d}$ is the $d$-dimensional Newton constant. Let us consider a black hole as a $d$-dimensional cube of size equal to twice its radius (Schwarzschild radius) $r_{\mathrm{s}}$; the uncertainty in the position of a Hawking particle, during the emission, is

$$
\Delta x \approx 2 r_{\mathrm{s}}=2 \lambda_{d}\left(\frac{G_{d} M}{c^{2}}\right)^{1 / d-3},
$$

where

$$
\lambda_{d}=\left[\frac{16 \pi}{(d-2) \Omega_{d-2}}\right]^{1 / d-3} .
$$

Using the usual uncertainty principle, the uncertainty in the energy of the Hawk- 
ing particle is

$$
\Delta E \approx c \Delta p \approx \frac{c}{\Delta x} \approx \frac{M_{\mathrm{pl}} c^{2}}{2 \lambda_{d}} m^{-1 /(d-3)},
$$

where $m=M / M_{\mathrm{pl}}$ is the mass in the Planck unit, $M_{\mathrm{pl}}$ is the $d$-dimensional Planck mass. $\Delta E$ is identified with the temperature of black hole radiation. Setting the black hole radiation mass $m$ to $\frac{d-3}{4 \pi}$, it is easy to obtain the temperature of the black hole in $d$-dimensional space-time. The Hawking temperature is related to the Schwarzschild radius by

$$
T=\frac{1}{4 \pi r_{\mathrm{h}}}=\frac{1}{2 \pi \Delta x} \text {. }
$$

From (3) and (4) we obtain

$$
T=\frac{d-3}{4 \pi \lambda_{d}} M_{\mathrm{pl}} c^{2} m^{-1 /(d-3)} .
$$

The evaporation of black hole would leave very distinctive imprints on the detectors and temperature of such black hole could be calculated. To study the quantum gravity effects in the Hawking temperature, one can take into account the generalized uncertainty principle. Generalized uncertainty principle has been the subject of much interesting works over the years and a lot of papers have appeared in which that usual uncertainty is modified at the framework of microphysics as $[13,14]$ :

$$
\Delta x_{i} \geq \frac{\hbar}{\Delta p_{i}}+l_{\mathrm{pl}}^{2} \frac{\Delta p_{i}}{\hbar},
$$

where $l_{\mathrm{pl}}$ is the Planck length. The term $l_{\mathrm{pl}}^{2} \frac{\Delta p_{i}}{\hbar}$ in Eq. (6) shows the gravitational effects to usual uncertainty principle. In the canonical quantum gravity the area of black hole factory is quantized as $A=n \alpha \hbar$ (with $G=c=1$ ). For this reason we must obtain the lower bound on the black hole factory radius. Let us consider a quantum black hole factory, an attempt to measure the radius of the black hole, more precisely that is, to make $R$ small - thus resulting in an increase in $\Delta p$, but according to Eq. (6) for detection of small distances by going to very high momenta, the behavior of the Heisenberg microscope changes and a lower bound on the (Schwarzschild) radius $r_{\mathrm{s}}$ could be obtained. Setting $2 r_{\mathrm{s}}$ as $\Delta x_{i}$ and inverting Eq. (6) we obtain

$$
\frac{r_{\mathrm{s}}}{2 l_{\mathrm{pl}}^{2}}\left(1-\sqrt{1-\frac{4 l_{\mathrm{pl}}^{2}}{r_{\mathrm{s}}^{2}}}\right) \leq \frac{\Delta p_{i}}{\hbar} \leq \frac{r_{\mathrm{s}}}{2 l_{\mathrm{pl}}^{2}}\left(1+\sqrt{1-\frac{42 l_{\mathrm{pl}}^{2}}{r_{\mathrm{s}}^{2}}}\right) .
$$

Comparing Eqs. (2), (4), (5) with (7) we obtain the Hawking radiation of $d$-dimensional black hole,

$$
T^{\prime}=\frac{d-3}{4 \pi} \lambda_{d} m^{1 /(d-3)}\left(1-\sqrt{1-\frac{1}{\lambda_{d}^{2} m^{2 / d-3}}}\right) M_{\mathrm{pl}} c^{2} .
$$

The Bekenstein-Hawking entropy is usually derived from the Hawking temperature. $M$ means energy and $T_{\mathrm{H}}$ means temperature. The entropy $s$ may be found 
from the well known thermodynamics relation,

$$
T=\frac{\mathrm{d} E}{\mathrm{~d} T}=\frac{\mathrm{d} M}{\mathrm{~d} T} \text {. }
$$

Inserting (8) to (9) one finds

$$
\frac{d-3}{4 \pi} \lambda_{d} m^{1 /(d-3)}\left(1-\sqrt{1-\frac{1}{\lambda_{d}^{2} m^{2 / d-3}}}\right) M_{\mathrm{pl}} c^{2}=\frac{\mathrm{d} M}{\mathrm{~d} S} .
$$

That means

$$
\begin{aligned}
S= & 2 \pi \lambda_{d}\left(\frac{1}{2} \ln \left|\frac{\sqrt{1-\frac{1}{\lambda_{d}^{2} m^{-2 / d-3}}}+1}{\sqrt{1-\frac{1}{\lambda_{d}^{2} m^{-2 / d-3}}}-1}\right|-1-\sqrt{1-\frac{1}{\lambda_{d}^{2} m^{-2 / d-3}}}\right. \\
& -\ln \mid 1-\sqrt{\left.1-\frac{1}{\lambda_{d}^{2} m^{-2 / d-3}} \mid\right)+ \text { const. }}
\end{aligned}
$$

Recalling that the Schwarzschild radius is given by

$$
r_{\mathrm{s}}=\lambda_{d}\left(\frac{G_{d} M}{c^{2}}\right)^{1 / d-3}
$$

the surface area of the black hole horizon is given by

$$
4 \pi\left(r_{\mathrm{s}}\right)^{2}=4 \pi \lambda_{d}\left(\frac{G_{d} M}{c^{2}}\right)^{1 / d-3}=\text { Area } .
$$

The fact that the entropy depends on the surface area rather than the volume of the black hole is reminiscent of the elementary physical fact that the total charge of an electrically charged solid sphere of material is related to the surface area, not the volume. So the black hole entropy is

$$
\begin{aligned}
S= & \frac{\text { Area }}{2}\left(\frac{G_{d} M_{\mathrm{pl}} m}{c^{2}}\right)^{-1 / d-3}\left(\frac{1}{2} \ln \left|\frac{\sqrt{1-\frac{1}{\lambda_{d}^{2} m^{-2 / d-3}}}+1}{\sqrt{1-\frac{1}{\lambda_{d}^{2} m^{-2 / d-3}}}-1}\right|-1\right. \\
& -\sqrt{1-\frac{1}{\lambda_{d}^{2} m^{-2 / d-3}}}-\ln \mid 1-\sqrt{\left.1-\frac{1}{\lambda_{d}^{2} m^{-2 / d-3}} \mid\right)+ \text { const. }}
\end{aligned}
$$

Equation (14) is the entropy of a higher dimensional black hole whose temperature is modified based on the generalized uncertainty principle.

\section{Conclusion}

The Bekenstein-Hawking entropy was derived from the Hawking temperature. The Hawking temperature receives a modification based on the generalized uncertainty principle. We presented a solution that shows the Bekenstein-Hawking entropy in the higher dimensional space. Furthermore, the thermodynamics of such system is studied. 


\section{Acknowledgments}

We thank the referee for helpful comments.

\section{References}

[1] N. Arkani-Hamed, S. Dimopoulos, G. Davali, Phys. Lett. B 429, 263 (1998).

[2] I. Antoniadis, N. Arkani-Hamed, S. Dimopoulos, G. Davali, Phys. Lett. B 436, 257 (1998).

[3] L. Randall, R. Sundrum, hep-ph/9905221.

[4] L. Randall, R. Sundrum, hep-th/9906064.

[5] P.C. Argyres, S. Dimopoulos, J. March-Russel, Phys. Lett. B 441, 96 (1998).

[6] A. Chamblin, S.W. Hawking, H.S. Reall, hep- th/9909205.

[7] B. Giddings, S. Thomas, Phys. Rev. D 67, 056010 (2002); S. Dimopoulos, G. Landsberg, Phys. Rev. Lett. 87, 161602 (2001); L.A. Anchordoqui, J.L. Feg, H. Goldberg, A.D. Shapere, Phys. Rev. D 67, 124027 (2002); S.B. Giddings, in: Future of Theoretical Physics and Cosmology, Eds. G.W. Gibbons, E.P.S. Shellard, S.J. Renkin, Cambridge University Press, Cambridge 2003.

[8] J.D. Beckenstein, Lett. Nuovo, Cimento 4, 737 (1972).

[9] J.D. Beckenstein, Phys. Rev. D 7, 2333 (1973).

[10] J.D. Beckenstein, Phys. Rev. D 9, 3292 (1974).

[11] S.W. Hawking, Commun. Math. Phys. 25, 152 (1972).

[12] J.M. Bardeen, B. Carter, S.W. Hawking, Commun. Math. Phys. 31, 161 (1973).

[13] R.J. Adler, P. Chen, D. Santiago, Gen. Rel. Grav. 33, 2101 (2001); M. Cavaglia, S. Das, hep-th/0404050; S. Das, hep-th/0403202; R.K. Kaul, P. Majumdar, Phys. Rev. Lett. 56, 5255 (2000); S. Carlip, Class. Quant. Grav. 17, 4175 (2000); S. Das, P. Majumdar, R.K. Bhaduri, Class. Quant. Grav. 19, 2355 (2002); S. Das, P. Majumdar, Phys. Rev. D 63, 044019 (2001); T.R. Govindarajan, R.K. Kaul, V. Suneeta, Class. Quant. Grav. 18, 2877 (2001); S. Mukhreji, S.S. Pal, JHEP 0205, 026 (2002); J.E. Lidsey, S. Nojiri, S.D. Odintsov, S. Ogushi, Phys. Lett. $B$ 544, 337 (2002); S. Nojiri, S.D. Odintsov, S. Ogushi, Int. J. Mod. Phys. A 18, 3395 (2003); E. Keski-Vakkuri, P. Kraus, Phys. Rev. D 54, 7407 (1996); M.K. Parikh, F. Wilczek, Phys. Rev. Lett. 85, 5042 (2000); S. Hemming, E. KeskiVakkuri, Phys. Rev. D 64, 044006 (2001); A. Farmany, S. Abbasi, A. Naghipour, Phys. Lett. B 650, 33 (2007), ibid, Erratum 659, 913 (2008).

[14] D. Amati, M. Ciafaloni, G. Veneziano, Phys. Lett. B 216, 41 (1989); D. Amati, M. Ciafaloni, G. Veneziano, Nucl. Phys. B 347, 550 (1990); M. Maggiore, Phys. Rev. D 49, 5184 (1994); Phys. Lett. B 319, 83 (1993); G. Veneziano, Europhys. Lett. 2, 199 (1986); E. Witten, Phys. Today 49, 24 (1996); R.J. Adler, D.I. Santiago, Mod. Phys. Lett. A 14, 1371 (1999); A. Farmany, EJTP 3, 12 (2006). 